\title{
LncRNA Gas5 acts as a ceRNA to regulate PTEN expression by sponging miR-222-3p in papillary thyroid carcinoma
}

\author{
Xiao-Fang Zhang ${ }^{1}$, Yan Ye $^{1}$ and Shu-Jun Zhao ${ }^{1}$ \\ ${ }^{1}$ Key Laboratory of Hormones and Development (Ministry of Health), Tianjin Key Laboratory of Metabolic Diseases, Tianjin \\ Metabolic Diseases Hospital and Tianjin Institute of Endocrinology, Tianjin Medical University, 300070 Tianjin, China \\ Correspondence to: Shu-Jun Zhao, email: zhaoshj@tijmu.edu.cn \\ Keywords: Gas5; competing endogenous RNA; PTEN; papillary thyroid carcinoma; proliferation \\ Received: June 03, $2017 \quad$ Accepted: November 26, $2017 \quad$ Published: December 16, 2017 \\ Copyright: Zhang et al. This is an open-access article distributed under the terms of the Creative Commons Attribution License 3.0 \\ (CC BY 3.0), which permits unrestricted use, distribution, and reproduction in any medium, provided the original author and source \\ are credited.
}

\section{ABSTRACT}

Accumulating evidence demonstrates that the long non-coding RNA Growth ArrestSpecific 5 (Gas5) has practical significance in cancer progression and metastasis. However, its role and function in papillary thyroid carcinoma (PTC) remains unknown. In this study, we aimed to explore the potential involvement of Gas5 in papillary thyroid carcinogenesis and to highlight the emerging roles of ceRNAs in the biological regulation of PTC cells. The results suggested that Gas5 was markedly downregulated in both PTC tissues and PTC cell lines. Over-expression of Gas5 remarkably suppressed PTC cells proliferation in vitro and inhibited the growth of tumor cells in vivo likewise. Furthermore, Gas5 was identified as a target of miR-222-3p which was aberrantly high in PTC cells. Enhanced expression of miR-222-3p promoted the proliferation of PTC cells while knocking down miR-222-3p could inhibit it. The advanced effects of miR-222-3p on the proliferation of PTC cells could be partly reversed by the upregulation of Gas 5 expression. Furthermore, we validated that Gas5 increased the protein level of the PTEN, one of miR-222-3p's targets, which further activated PTEN/AKT pathway. Taken together, our study identified a tumor suppressive role of Gas5 in PTC cells acting as a ceRNA, effectively becoming a sink for miR-222-3p, modulating the expression of PTEN, which lead to PTEN/AKT pathway activation and proliferation suppression. This finding may offer a new potential therapeutic strategy for PTC.

\section{INTRODUCTION}

Thyroid cancer, with an increasing incidence in recent years, is the most common type of endocrine malignancies [1-3]. It is classified into four histologic types: papillary, follicular, medullary, and anaplastic thyroid carcinoma. Papillary thyroid carcinoma (PTC) is the main form of thyroid cancer, accounting for greater than $80 \%$ of all thyroid malignancies mainly in young women and children [4]. Although PTC has a favorable prognosis for patients in early stages, in which the overall five-year survival rate is $97 \%$, patients with advanced thyroid cancer only have a five-year survival rate of about $59 \%$ [5]. Therefore, it is essential to identify more effective therapeutic strategies.

Recently, people have paid more attention on long non-coding RNAs (lncRNAs). As a new modulator,
IncRNAs have been shown to regulate tumor progression [6]. Numerous data have showed that lncRNAs (defined as $>200 \mathrm{nt}$ ) are dysregulated in cancer biology [7]. In some pathological processes, such as cancer, the expression levels of $\operatorname{lncRNAs}$ are increased (e.g., HOTAIR) or decreased (e.g., Gas5) [8-10]. Once a report demonstrated that dysregulated expressions of key lncRNAs in ceRNA (competing endogenous RNA) network focused on the miRNA-mediated lncRNA/mRNA crosstalk and broke bistable condition [11]. Gas5 (Growth Arrest-Specific 5) has been first observed by a study that demonstrated a tumor suppression role in 1988. Since then, Gas5 became a star gene among researchers [12, 13]. Accumulating data showed that Gas5 could be implicated in the tumorigenesis and progression of many cancers and may be a useful diagnostic and prognostic cancer biomarker [14]. However, to date, the role of Gas5 in PTC remains 
largely unexplored. Therefore, the present study aims to explore the potential role of Gas5 in papillary thyroid carcinogenesis and to highlight the emerging roles of ceRNAs in the biological regulation of PTC cells.

PTEN (phosphatase and tension homologue) has been identified as a tumor suppressor gene to be involved in the multi-step biological processes, such as focal adhesion, migration, and proliferation of cancer cells $[15,16]$. MiRNAs were reported deregulated and promoted cancer development by down-regulating PTEN expression in some cancers [17]. Among them, the expression of miR-222-3p and its target gene PTEN, as well as their relationship has been established in PTC. Low level of PTEN activity induces phosphorylation of AKT, promoting cell proliferation and migration [18-20].

In this study, we verified the tumor suppressive role of Gas5 as a ceRNA in PTC to modulate PTEN level and identified miR-222-3p as the specific miRNA decoyed by Gas5. Furthermore, we highlighted that Gas5 activated PTEN/AKT pathway repressing the proliferation of PTC cells.

\section{RESULTS}

\section{Gas5 was down-regulated in papillary thyroid carcinoma (PTC) tissue specimens and PTC cell lines}

Numerous evidence links Gas5 dysregulation to human cancers. To verify whether the expression of Gas5 is reduced in PTC clinical tissue specimens, we conducted a review of previous microarray data for the expression of Gas5 on mRNA level in human PTC. Results of researches in Oncomine database (www.oncomine.com) (Rhodes et al., 2004) (Figure 1A) revealed that expression of Gas5 was markedly decreased in human PTC as compared with healthy thyroid tissues as shown in He datasets (Proc Natl Acad Sci U S A 2005) ( $p=0.008)$.

Furthermore, to confirm the reduced expression of Gas5 in PTC cell lines, we performed qRT-PCR analysis to detect the Gas5 mRNA expression in 4 PTC cell lines (BHP5-16, TPC, K1, BHP2-7) and a normal human thyroid cell line Nthy-ori 3-1. As illustrated in Figure 1B, Gas5 mRNA expression level was significantly lower in four PTC cell lines as compared with Nthy-ori 3-1 $(P<0.01$, respectively).

These results provide a strong support for the idea that Gas5, which is commonly decreased in many cancers and associated with clinic-pathological characteristics, is frequently reduced in PTC.

\section{Gas5 represses proliferation of PTC cells in vitro and in vivo}

The remarkable decrease of Gas5 expression in PTC tissue samples and PTC cell lines stimulated us to evaluate the possible mechanism of Gas5 in pathological process of PTC.

To enhance Gas5 expression in PTC cells, a pcDNA3.1/Gas5 vector was constructed and transfected into $\mathrm{BHP} 5-16$ and $\mathrm{K} 1$ respectively. The cells transfected with pcDNA3.1 plasmid were acted as a control. QRT-PCR analysis of Gas5 mRNA levels was performed at $48 \mathrm{~h}$ post-transfection and revealed that Gas5 expression was increased 5.4-fold in BHP5-16 cells and 4.2-fold in K1 cells, as compared with control group respectively (Figure 2A). Then MTT and colony formation assays were performed to investigate the function of Gas5 on cell proliferation in vitro. In MTT assays, results showed that the cell
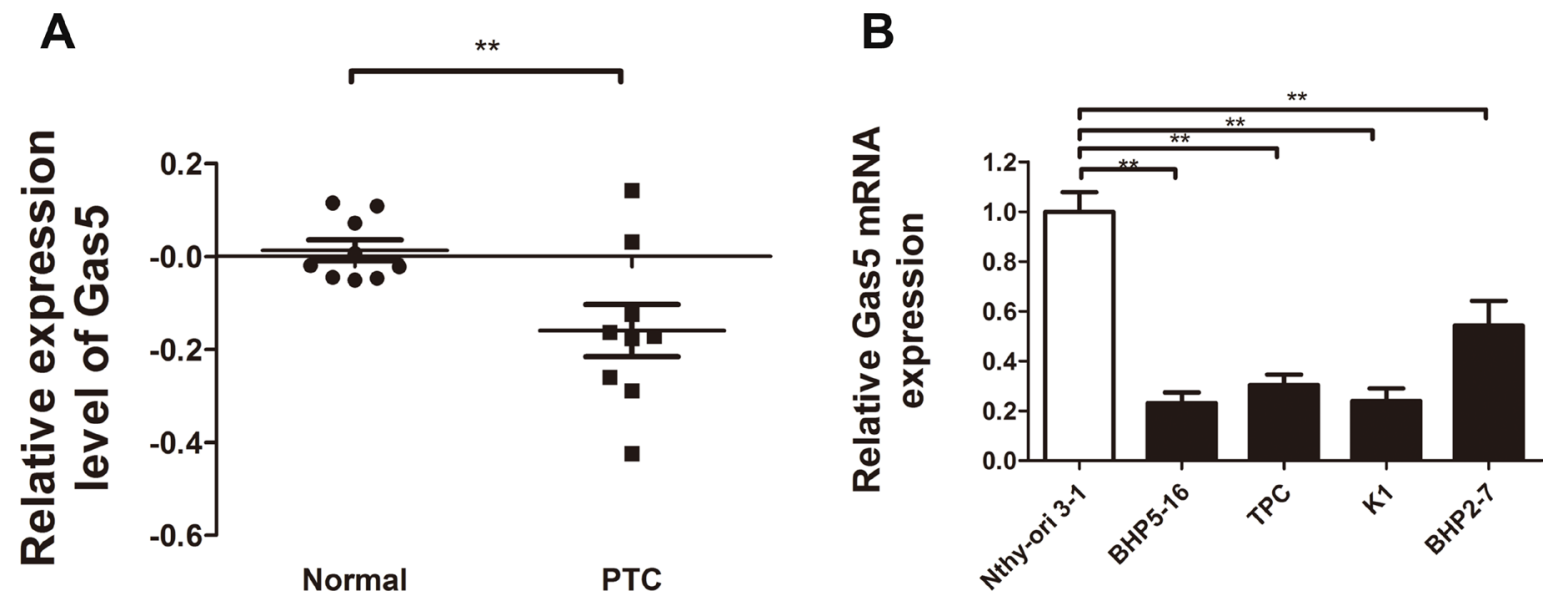

Figure 1: Relative Gas5 expression in papillary thyroid carcinoma (PTC) tissue specimens and PTC cell lines. (A) Gas5 expression was significantly decreased in human PTC tissue specimens as compared with healthy thyroid tissues $(P=0.008)$. Nine paired thyroid gland papillary carcinoma and normal thyroid gland samples were analyzed in He datasets (Proc Natl Acad Sci U S A 2005). (B) Quantitative RT-PCR analysis of Gas5 in PTC cell lines (BHP5-16, TPC, K1, BHP2-7) compared with a normal human thyroid cell line Nthy-ori3-1. ${ }^{* *} P<0.01$, compared with control. 
proliferation of BHP5-16 and K1 were significantly decreased in pcDNA3.1/Gas5 transfected cells compared with the control respectively (Figure 2B). Likewise, the results of colony formation assays showed that clonogenic survival was dramatically decreased following forced expression of Gas5 in both BHP5-16 and $\mathrm{K} 1$ cells (Figure 2C). To explore whether the level of Gas 5 expression affects tumorigenesis, BHP5-16 cells transfected with pcDNA3.1/Gas5 or pcDNA3.1 were used in xenograft model of the nude mice. Up to 6 weeks after
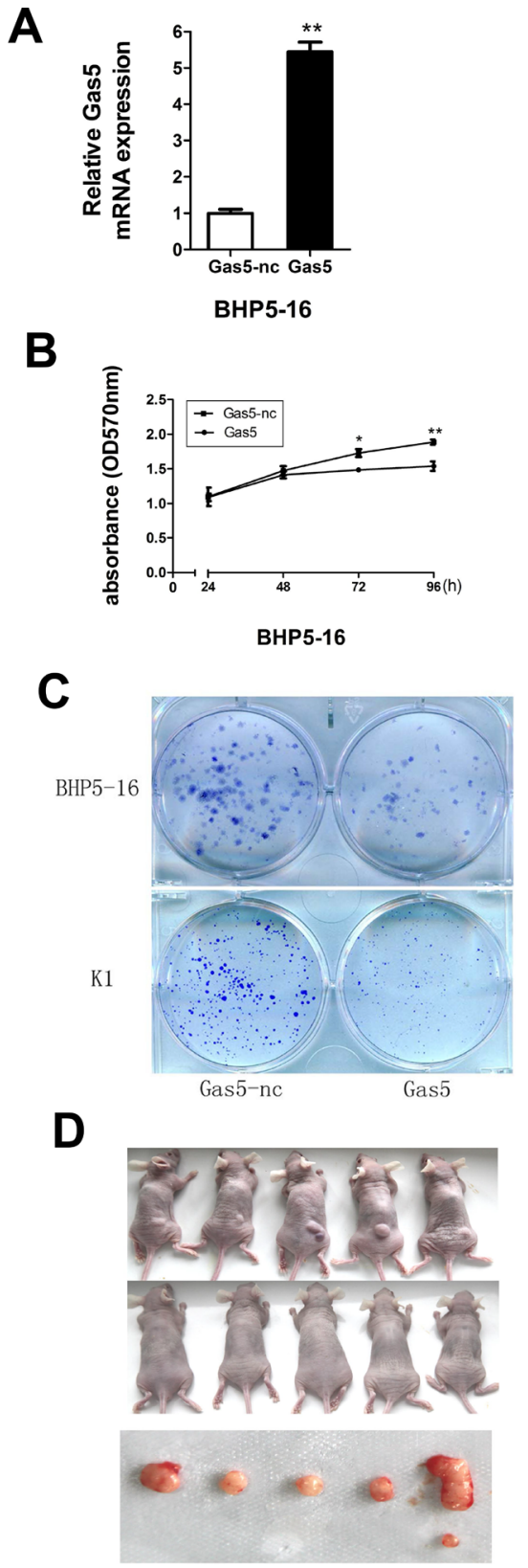

injection, tumors were found in all of mice in pcDNA3.1 group while only one mouse bore a tumor in pcDNA3.1/ Gas5 group. The tumor volume of pcDNA3.1/Gas5 group is dramatically decreased compared with pcDNA3.1 group (Figure 2D).

In summary, these results show that forced expression of Gas 5 can repress proliferation capacity of PTC cells not only in vitro but also in vivo. Nevertheless, the detailed mechanism by which Gas 5 functions need to be further investigated.
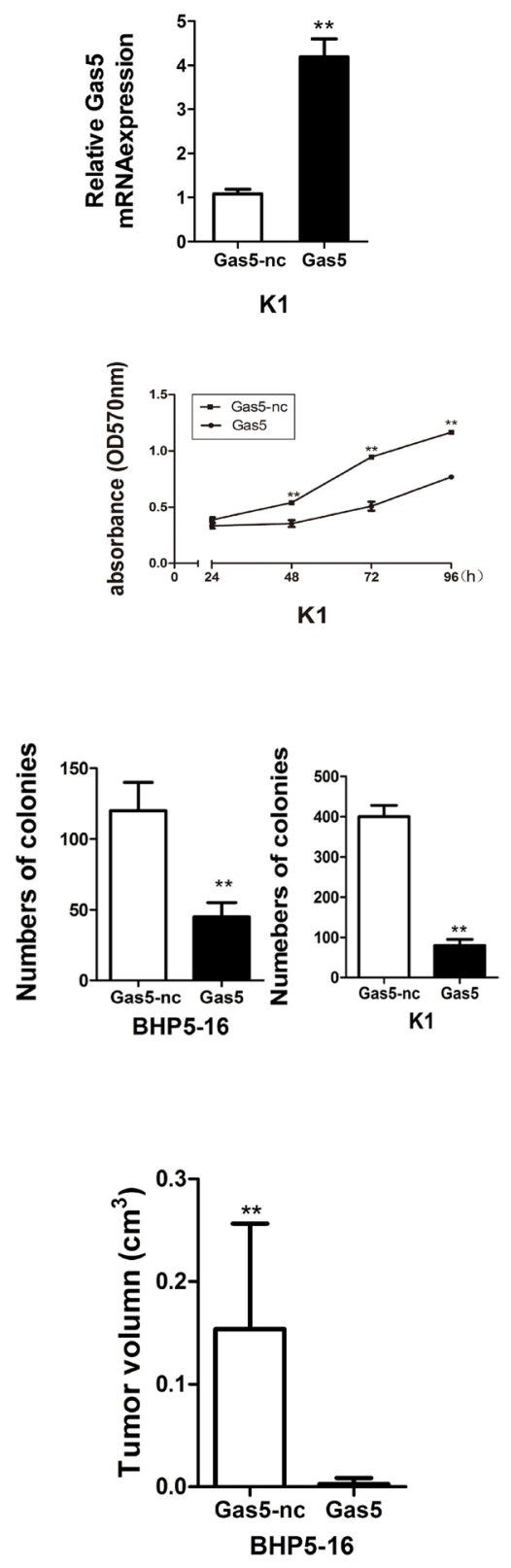

Figure 2: Gas5 represses proliferation of PTC cells in vitro and in vivo. (A) QRT-PCR analysis of Gas5 expression level following treatment BHP5-16 and K1 cells with pcDNA3.1/Gas5 vector. (B) The proliferation of pcDNA3.1/Gas5 vector-transfected BHP5-16 and K1 cells were detected by MTT assay. (C) Colony formation assay was performed to determine the proliferation of pcDNA3.1/Gas5 vector-transfected BHP5-16 and K1 cells. Colonies were counted and captured. Data represent the mean \pm SD from three independent experiments. (D) Tumor formation assay was conducted in female BALB/c nude mice by subcutaneous injection of BHP5-16 cells $\left(5 \times 10^{5}\right)$ transfected with pcDNA3.1/Gas5 or pcDNA3.1 into the back of the mice. After 6 weeks, tumor tissues were harvested and tumor volumes were measured. ${ }^{* *} P<0.01$ compared with control. 


\section{Gas5 is a target of miR-222-3p}

Previous reports demonstrated that lncRNA might act as a ceRNA to miRNA [21]. But accurate regulatory mechanisms of Gas5 remains unclear. Bioinformatics analysis (Starbase 2.0, RNA22) of miRNA recognition sequences on Gas5 revealed the presence of more than 30 miRNAs binding sites. Among them, miR-222-3p stood out through detailed survey, which was considered to facilitate tumor accelleration effect in PTC [22, 23]. Meanwhile, we detected the expression level of endogeneous miR-222-3p in BHP5-16 and K1 cells by qRT-PCR and the results demonstrated that it was drastically increased in both cell lines compared with Nthy-ori 3-1 cell (Figure 3A). Starbase 2.0 and RNA22 predictions revealed that the 3'-UTR of Gas 5 mRNA contained two miR-222-3p binding sites (Figure $3 \mathrm{~B}(\mathrm{a})$ ). And then, to experimentally confirm that Gas5 was a target of miR-222-3p, we constructed two recombinant luciferase reporter vectors of Gas5 3'-UTR, i.e., RLuc-Gas5-3'-UTR-WT and RLuc-Gas5-3'-UTRMT. The recombinant luciferase mRNA transcribed by RLuc-Gas5-3'- UTR-WT carried all miR-222-3p binding sites (Gas5-3'-UTR-WT) predicted in Gas5 3'-UTR while the one transcribed by RLuc-Gas5-3'-UTR-MT lacked the predicted binding site 1 (Gas5-3'-UTR-MT) (Figure 3B(b)). Considering the low value of binding energy predicted by software, we didn't mutate the second binding site. Cotransfection of RLuc-Gas5-3'-UTR-WT and miR-222-3p mimics was conducted in both BHP5-16 and K1 cells. Co-transfection with RLuc-Gas5-3'-UTR-WT and miR-nc served as a control. The results demonstrated that luciferase activity was reduced by nearly $20 \%$ in miR- $222-3$ p cotransfection group compared with the control. To further confirm that the decrease of luciferase activity in the RLucGas5-3'-UTR-WT vector co-transfected cells was due to direct interaction between the miR-222-3p and its putative binding site, we transfected BHP5-16 and K1 with RLucGas5-3'-UTR-MT (lacking the predicted binding site 1) (Gas5-3'-UTR-MT) and miR-222-3p mimics to monitor the luciferase activity. The results revealed that the suppression of luciferase activity was completely abolished in RLucGas5-3'-UTR-MT and miR-222-3p mimics co-transfection cells (Figure 3C).

Taken together, these data suggest that miR-222$3 p$ can directly bind to Gas 5 through miRNA recognition sites. As a target of miR-222-3p, Gas5 may act as a ceRNA to $\mathrm{miR}-222-3 \mathrm{p}$.

\section{MiR-222-3p promotes proliferation of PTC cells in vitro}

By the analysis of the genome-wide miRNAs expression profile in human papillary thyroid carcinoma (PTC), researchers found an over-expression of miR146b-5p, miR-2223-p and some other miRNAs in PTCs that clearly differentiates PTCs from normal thyroid tissues $[22,23]$. To investigate the role of miR-222-3p on PTC carcinogenesis, miR-222-3p mimics or inhibitor was transfected into both BHP5-16 and K1 cells and the proliferation curves were performed using MTT assays. Our results showed that over-expression of miR-222-3p markedly promoted the cell growth in both BHP5-16 and $\mathrm{K} 1$ cells when compared with cells transfected with miR-nc. Conversely, the cells transfected with miR-222-3p inhibitor grew at a dramatically lower rate as compared with controls (Figure 4A). Also, colony formation capacity was detected in miR-222-3p mimics, inhibitor or miR222-3p mimics plus pcDNA3.1/Gas5 treated K1 cells. We observed an enhanced colonigenic capacity in miR-222-3p over-expression cells as compared with controls, which could be reversed by Gas5 co-transfection. By contrast, a dramatically reduction of colony forming capacity has been demonstrated in miR-222-3p inhibitor transfected cells as compared with controls (Figure 4B).

Collectively, these data indicate that miR-222-3p can accelerate PTC cells proliferation, which inversely correlates with the effects of Gas5 in PTC cells.

\section{Gas5 controls the miR-222-3p's target, PTEN}

Among the many predicted targets of miR-222$3 p$ by bioinformatics software (TargetScan, MiRanda), we focused on PTEN. PTEN is known to act as a tumor suppressor protein, which inhibits the PI3K/AKT pathway, and regulating cell processes including growth, proliferation and polarization [24]. The predicted binding site of miR-222-3p on the $3^{\prime}$-UTR of PTEN mRNA is shown in Figure 5A(a). And then, to experimentally confirm that PTEN was a target of miR-222-3p, we constructed two recombinant luciferase reporter vectors of PTEN 3'-UTR, i.e., RLuc-PTEN-3'-UTR-WT and RLucPTEN-3'-UTR-MT. The recombinant luciferase mRNA transcribed by RLuc-PTEN-3'-UTR-WT carried all miR222-3p binding sites (PTEN-3'-UTR-WT) predicted in PTEN 3'-UTR while the one transcribed by RLuc-PTEN3'-UTR-MT lacked the predicted binding site (PTEN-3'UTR-MT) (Figure 5A(b)).

Co-transfection of RLuc-PTEN-3'-UTR-WT and miR-222-3p mimics was conducted in both BHP5-16 and K1 cells. Co-transfection with RLuc-PTEN-3'UTR-WT and miR-nc served as a control. The results of dualluciferase assay system demonstrated that the activity was reduced by nearly $30 \%$ in miR-222-3p cotransfection group compared with the control. To provide further evidence that the decrease of luciferase activity in the RLuc-PTEN-3'-UTR-WT vector co-transfected cells was due to direct interaction between the miR-222$3 \mathrm{p}$ and its putative binding site, we transfected BHP516 and K1 with RLuc-PTEN-3'-UTR-MT (lacking the predicted binding site) (PTEN-3'-UTR-MT) and miR-222$3 \mathrm{p}$ mimics to monitor the luciferase activity. The results revealed that the suppression of luciferase activity was completely eliminated in RLuc-PTEN-3'-UTR-MT and miR-222-3p mimics co-transfection cells, proving that 
the mutation of the target sites of PTEN 3'-UTR is able to block the function of miR-222-3p (Figure 5B). These results suggested that miR-222-3p, as predicted, binds directly to putative PTEN 3'-UTR regions. Furthermore, quantitative RT-PCR (qRT-PCR) and western blot analysis were also applied to monitor the expression level of PTEN in the cell lines transfected with miR-222-3p mimics or inhibitor. QRT-PCR showed that no significant difference of PTEN mRNA level was found in the miR-222-3p mimics transfected cells as compared with cells transfected with miR-nc (Figure 5C). By western blot analysis, we found that up-regulation of miR-222-3p significantly decreased the expression of PTEN while down-regulation of miR-222-3p significantly increased the expression of PTEN (Figure 5D).
Taken together, our results confirm that miR-222-3p directly targets PTEN and represses its expression at posttranscriptional level in PTC cell lines, confirming the previous reports $[25,26]$.

Considering that Gas5 is a target of miR-222-3p, we deduce that Gas5 may serve as a 'sponges' or 'decoys' for miR-222-3p, modulating the expression of PTEN. Thus, the effect of Gas5 on the expression of PTEN protein was investigated. Western blotting analysis indicated that forced expression of Gas5 in BHP5-16 and $\mathrm{K} 1$ cells exerted a promoting role on endogenous PTEN protein expression (Figure 5E). In addition, we further observed the level of PTEN in cells co-transfected with Gas5 and miR-222-3p. The results showed that the PTEN
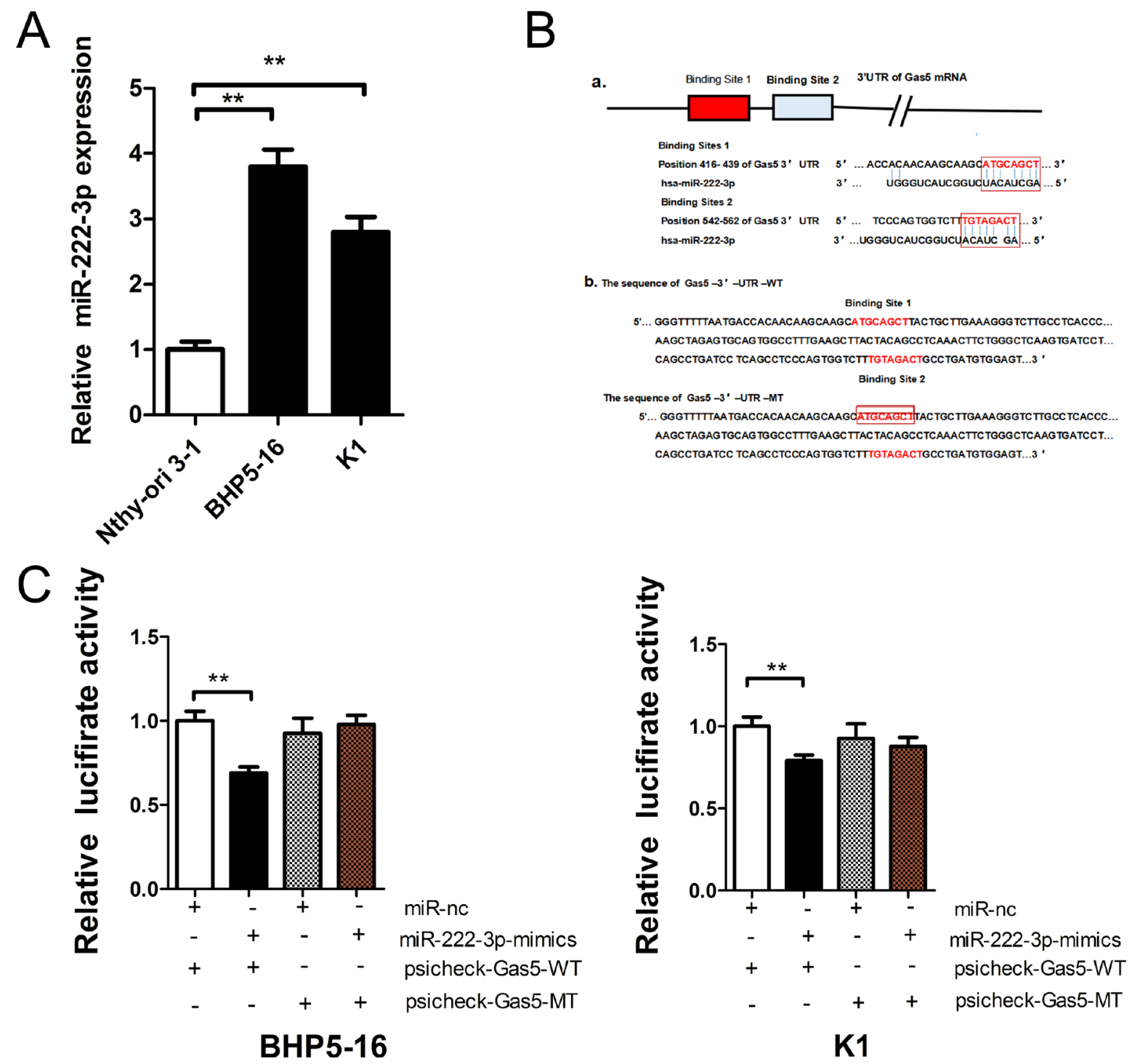

Figure 3: Gas5 is a target of miR-222-3p. (A) The expression level of endogeneous miR-222-3p in BHP5-16 and K1 cell. (B) a: Two miR-222-3p binding sites in Gas5 3'-UTR predicted with software. b: Wild (Gas5-3'-UTR-WT) and mutant (Gas5-3'-UTR-MT) Gas5 3'-UTRs carried in recombinant luciferase mRNAs transcribed by RLuc-Gas5-3'-UTR-WT and RLuc-Gas5-3'- UTR-MT. The binding site 1 was deleted from Gas5-3'-UTR-MT. (C) The luciferase reporter plasmid containing wild or mutant-type Gas5 3'-UTR was co-transfected into BHP5-16 and K1 cells with miR-222-3p mimics in parallel with miR-nc. Histogram indicate values of luciferase measured $48 \mathrm{~h}$ after transfection. Data represent the mean \pm SD from three independent experiments. ${ }^{* *} P<0.01$ compared with control. 
level of the group co-transfected with Gas5 and miR-222$3 p$ was much higher than that of the group transfected with miR-222-3p, while slightly lower than that of the Gas5 transfecting group with no statistical significance (Figure 5F). In conclusion, these data confirm that Gas5 can up-regulate the expression of PTEN in PTC cancer partly by mediate miR-222-3p and control its targets PTEN.
In order to demonstrate that Gas5 modulates the expression of PTEN partly by competitive binding to miR-222-3p in PTC cells, we carried out a luciferase assay. The plasmid pcDNA3.1/Gas5 or pcDNA3.1 was co-transfected with miR-222-3p mimics and RLuc-PTENWT 3'UTR into BHP5 -16 and K1 cells. Results showed that in the presence of Gas5, RLuc-PTEN-WT 3'-UTR repression was removed compared with the control
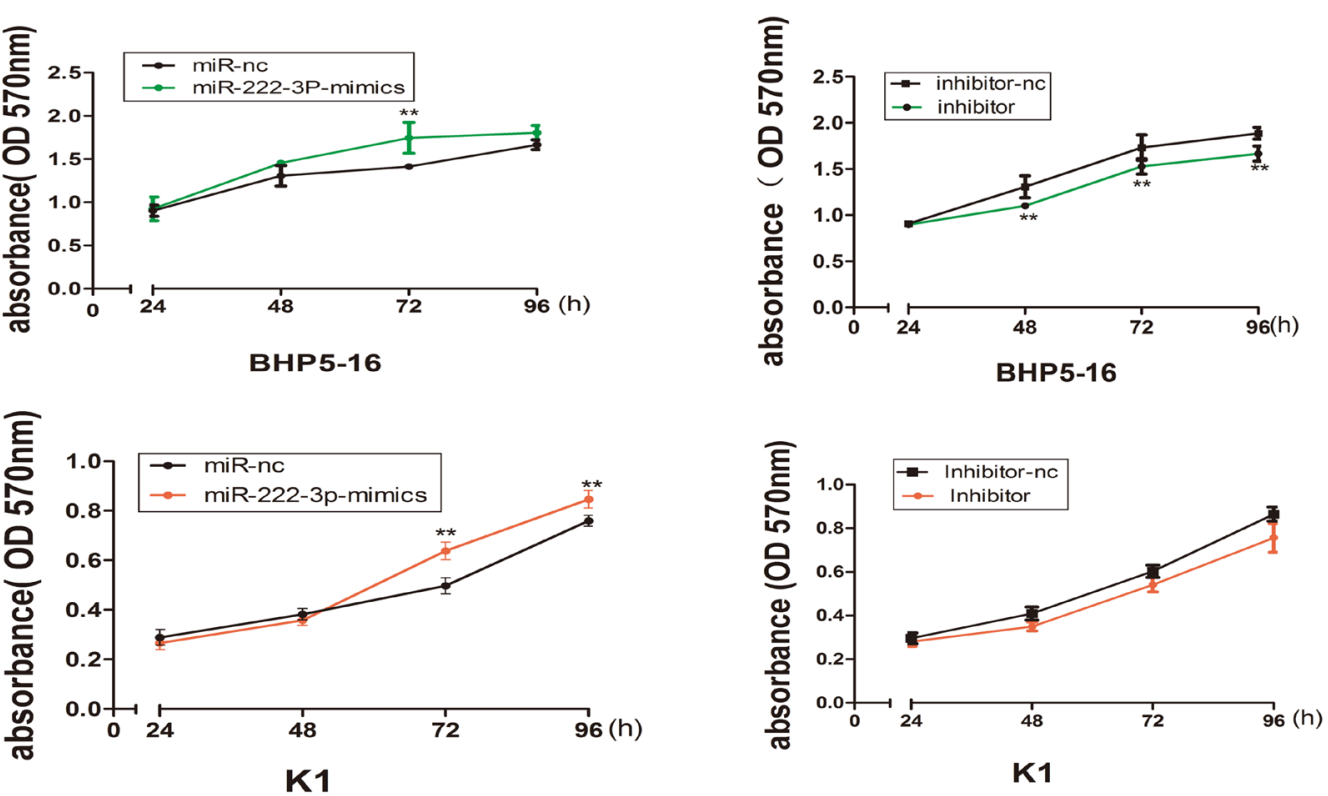

B

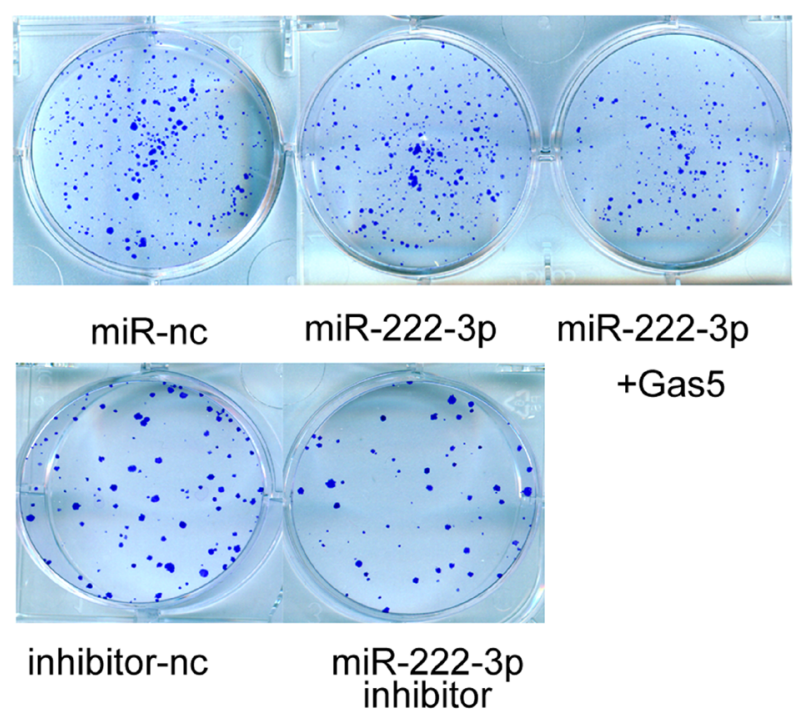

K1
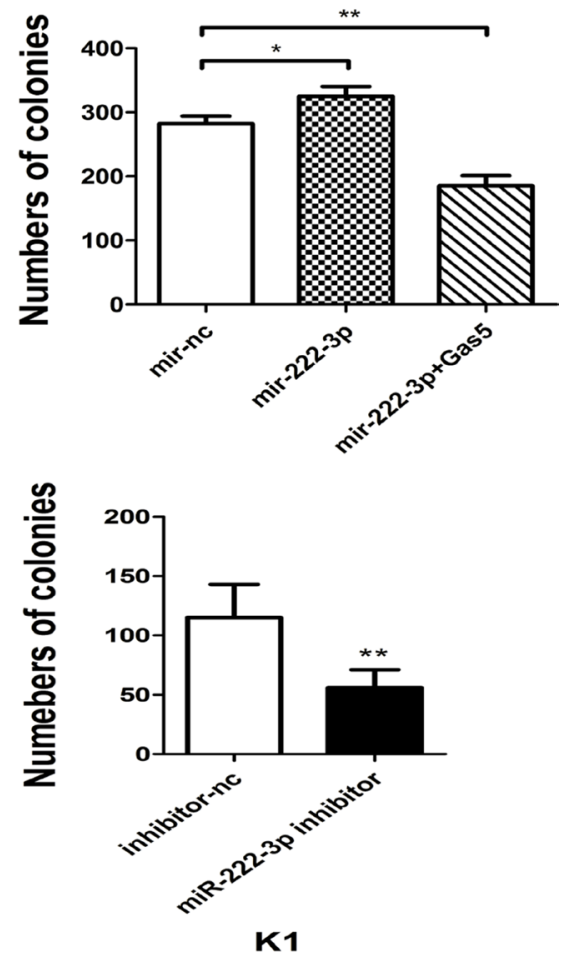

Figure 4: MiR-222-3p promotes proliferation of PTC cells in vitro. (A) Cell proliferation assay of BHP5-16 and K1 transfected with miR-222-3p mimics/inhibitor, the OD values were detected each day at the same time point by MTT assay. (B) Colony formation assays were performed to determine the proliferation of K1 cells transfected with miR-222-3p mimics, inhibitor or miR-222-3p mimics plus pcDNA3.1/Gas5. Data are expressed as mean $\pm \mathrm{SD}$ from three independent experiments. ${ }^{*} P<0.05,{ }^{* *} P<0.01$. 
group (Figure $5 \mathrm{G}$ ). These findings indicate that aberrant expression of Gas5, a ceRNA of miR-222-3p, may be involved in the progression of PTC by abolishing the miR222-3p induced repressing activity on PTEN.

\section{Gas5 activates PTEN/AKT pathway repressing the proliferation of PTC cells}

Next, we further investigated the mechanism of Gas5 in the regulation of the viability of PTC cells. The PI3K/ AKT signaling pathway exerts the effect on cell proliferation through its downstream effector AKT. PTEN is a key suppressor of oncogenic PI3K/AKT signaling. Considering the changes of expression level of PTEN, we detected the activity of the PI3K/AKT pathway in cells transfected with Gas5, miR-222-3p mimics, miR-222-3p inhibitor or Gas5 and miR-222-3p mimics respectively. Cells transfected with pcDNA3.1 or miR-nc was considered as the control. The western blot results showed the phosphorylation level of AKT (P-AKT). Just like knocking down miR-222-3p by miR-222-3p inhibitor, forced expression of Gas 5 decreased the level of P-AKT as compared with the control group. In contrast, over-expression of miR-222-3p enhanced P-AKT level, which could be reversed by Gas5. No statistical difference of total AKT level was found between the groups (Figure 5D-5F). These data demonstrated that Gas5 can activates PTEN/AKT pathway repressing the proliferation of PTC cells, which may be partly mediated by sponging miR-222-3p.

\section{DISCUSSION}

It is now increasingly acknowledged that lncRNAs might participate in the biological process of cancer. Therefore, detailed molecule mechanism of IncRNA may lead to new insights into pathogenetic process and novel therapy for this important disease [27]. LncRNA-Gas5 was a non-protein coding gene which was preferentially expressed in growth-arrested cells [28]. A large body of research suggested that Gas 5 was dysregulated in multiple cancers, such as prostate cancer and breast cancer [29-31] and confirmed a tumor suppressor role for this molecule. However, little is known regarding its expression and function in thyroid cancer.

In this research, we first conducted a review of previous microarray data in Oncomine database (www. oncomine.com) for the expression of Gas5 on mRNA level in human PTC. And then we examined the expression level of Gas5 in papillary thyroid carcinoma cells and identified the function of Gas5 by applying forced expression approaches. We confirmed that Gas5 was downregulated in papillary thyroid carcinoma clinical samples as well as in PTC cell lines, which was consistent with the recently reported findings of Guo et al. [32]. Over-expression of Gas5 significantly inhibited cell proliferation ability in vitro and in vivo manifested by MTT, colony formation assays and tumor formation assays. Thus, we may deduce that Gas5 may represent a potential biomarker and a new therapeutic target for PTC, which prompts us to explore the detailed mechanisms by which Gas5 inhibits thyroid carcinogenesis and PTC progression, stimulates novel research directions in PTC.

Sitimulated by the ceRNA regulatory circuitry and more and more data that IncRNAs may be involved in this regulatory network, we hypothesized that Gas5 may also act as a ceRNA exerting its biological function in PTC. To explore the correlation between miRNA and Gas5 in PTC pathogenesis, we employed bioinformatics analysis and found that the miR-222-3p had a higher score binding to Gas5, there followed a further study with a particular focus on the miR-222-3p's target gene PTEN. Luciferase assays of this case verified a novel ceRNA regulatory network, Gas5/miR-222-3p /PTEN. Consistent with Gas5 sequestration of miR-222-3p, we found that Gas5 overexpression restored PTEN protein synthesis in cells treated with miR-222-3p.

PTEN is a key suppressor gene of tumor cell growth by inhibiting the phosphorylation of AKT. In this study, dual-luciferase assay and western blot verified that PTEN was one target of miR-222-3p. Forced expression of miR$222-3 p$ by mimics transfecting significantly decreased the level of PTEN, accompanied by an remarkably increasing level of p-AKT while miR-222-3p knockdown by inhibitors showed an obviously opposite results. Previous researches reported that miR-222 played multiple roles in cancers acting as an onco-miR [33-35] or a tumor suppressor-miR [36, 37] depending on their target genes. In PTC, Pallante [38] reported that expression of miR221,-222 and-181b had 5-to 35- fold differential in FNAB samples of PTCs compared with other thyroid nodules. Furthermore, together with other miRNAs, miR-222 is considered as a signature that differentiates malignant from benign indeterminate thyroid lesions [39], the degree of aggressiveness of papillary thyroid carcinoma [40] or a circulating biomarker of recurrent papillary thyroid cancer [41]. In our study, we confirmed that miR-222-3p can promote the proliferation of PTC cells by targeting PTEN consequently activating PTEN/AKT pathway. At the same time, over-expression of Gas5 in PTC cells lead to a contrary result, that is, a higher level PTEN and a lower level p-AKT as compared with control. Furthermore, Gas5 can reverse the effects of miR-222-3p. Therefore, we may reasonably propose that Gas5, acting as a ceRNA, exerts a miRNA/lncRNA trans-regulatory effect on protein-coding mRNAs. Therefore, Gas5 may be a potential prognostic marker and therapeutic target.

However, it should be considered that the ceRNA activity of Gas5 may enable it to sponge a mass of miRNAs, while one miRNA is likely to regulate multiple genes as well. Therefore, the cellular phenotypes we observed are likely due to simultaneous targeting of multiple targets in PTC. Besides, there may be many 
A

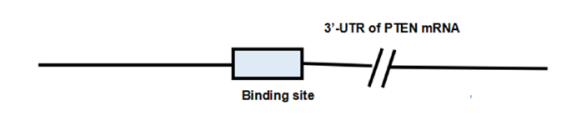

a. Binding site

Position 186-206 of PTEN 3. UTR $5^{*}$...UUGAAAAAAGGUUGUGUAGCU.... $3^{*}$

hsa-miR-222-3p

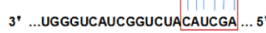

b. The sequence of PTEN -3'. UTR.WT

5... UUGaAaAaAgGuUguguagcu... $3^{*}$

The sequence of PTEN - -3. UTR-MT

5. ...UUGaAaAaAgGUUGUGUAGEG... 3 ,

C

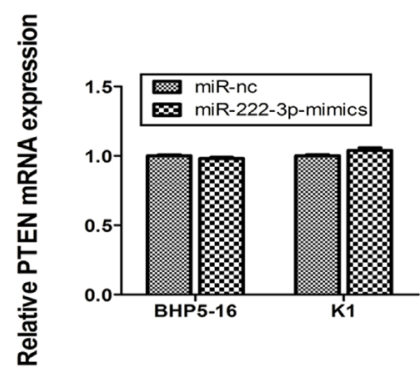

D

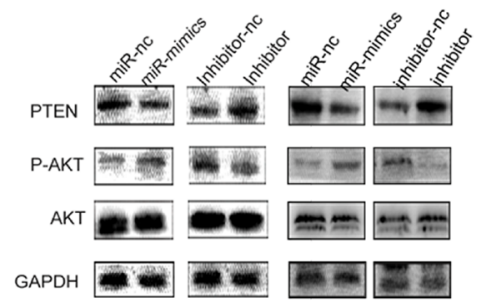

BHP5-16

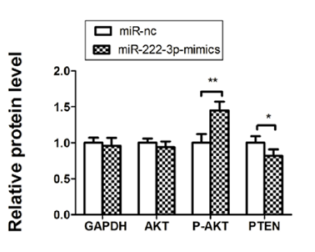

BHP5-16

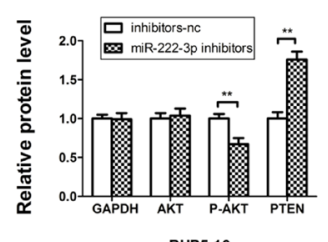

BHP5-16 k1

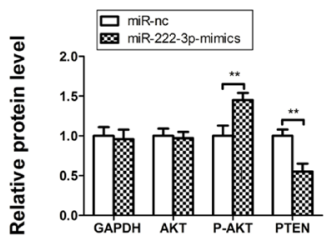

K1

K1

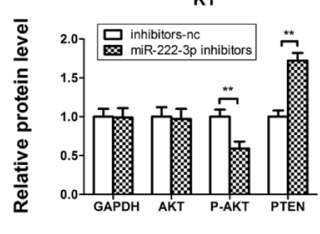

B

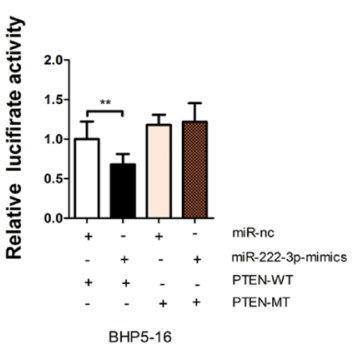

E

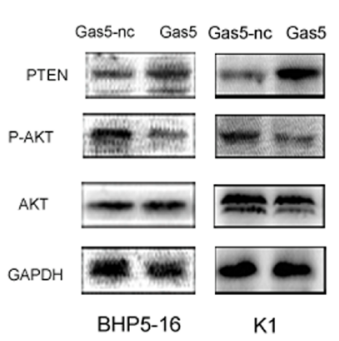

$\mathbf{F}$

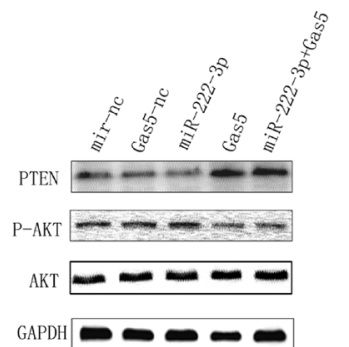

BHP5-16

G

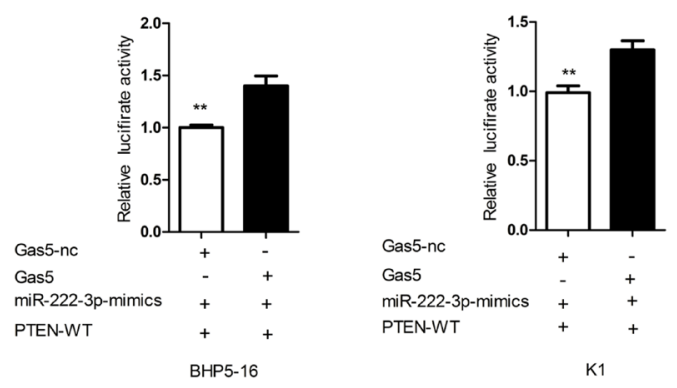

Figure 5: Gas5 controls the miR-222-3p's target, PTEN. (A) a: The binding site between miR-222-3p and the $3^{\prime}$-UTR of PTEN mRNA predicted by bioinformatics. b: Wild (PTEN-3'-UTR-WT) and mutant (PTEN-3'-UTR-MT) PTEN 3'-UTRs carried in recombinant luciferase mRNAs transcribed by RLuc-PTEN-3'-UTR-WT and RLuc-PTEN-3'- UTR-MT. The binding site was deleted from PTEN3'-UTR-MT. (B) The luciferase reporter plasmid containing wild or mutant-type PTEN-3'-UTR was co-transfected into BHP5-16 and K1 cells with miR-222 -3p mimics in parallel with miR-nc. (C) The mRNA level of PTEN in BHP5-16 and K1 cells transfected with miR-222-3p-mimics or miR-nc. (D) PTEN protein levels were detected by western blot in BHP5-16 and K1 cells after transfection with miR-222-3p mimics or miR-222-3p inhibitor. Meanwhile, the total and phosphorylated AKT were examined by western blot. GAPDH was used as an internal control. Similar results were obtained in three independent experiments. (E) Western blot analysis of PTEN, total AKT and phosphorylated AKT protein level following treatment of BHP5-16 cells and K1 with pcDNA3.1/Gas5 vector. GAPDH was used as control. (F) Western blot analysis of PTEN, total AKT and phosphorylated AKT protein level following treatment of BHP5-16 cells with pcDNA3.1/Gas5 vector and miR-222-3p mimics. (G) Rluc-PTEN 3'-UTR-WT and miR-222-3p mimics were co-transfected into BHP5-16 and K1 cells with plasmids expressing pcDNA3.1/Gas5 vector or with a control vector to verify the ceRNA activity of Gas5. Histogram indicate values of luciferase measured $48 \mathrm{~h}$ after transfection. Data represent the mean $\pm \mathrm{SD}$ from three independent experiments. ${ }^{* *} P<0.01$. 
other lncRNAs functioning as ceRNAs involved in the pathogenesis of PTC. Thus, the identification of these ceRNAs will ultimately promote the progression of lncRNA-directed diagnostics and treatment against PTC.

Taken together, our study provides experimental evidence that Gas5 functions as a competing endogenous RNA to regulate PTEN expression by sponging miR222-3p in papillary thyroid carcinoma (Figure 6). This finding may offer a new potential targeting therapy for the treatment of PTC.

\section{MATERIALS AND METHODS}

\section{Oncomine database analysis}

The informatics data on Gas 5 mRNA expression in papillary thyroid carcinoma were obtained from the Oncominedatabase (https://www.oncomine.org, Rhodes et al., 2004). He et al. (GEO accession GSE3467) datasets were used to compare the expression levels of Gas5 between PTC and normal thyroid tissues.

\section{Cell culture and reagents}

Four papillary thyroid carcinoma cell lines (BHP5-16, TPC, K1 and BHP2-7) and a normal human thyroid epithelium cell line (Nthy-ori 3-1) were used in this study. Among them, BHP5-16 and BHP2-7 were donated by Pro. Tenglisong from Zhejiang university, China. K1, TPC and
Nthy-ori 3-1 were donated by Pro. Yuyang from cancer hospital, Tianjin medical university, China. Cells were cultured in RPMI 1640 (Invitrogen) supplemented with $10 \%$ fetal bovine serum(Gibico) $100 \mathrm{U} / \mathrm{ml}$ penicillin, and $100 \mathrm{mg} / \mathrm{ml}$ streptomycin(Invitrogen) in humidified air at $37^{\circ} \mathrm{C}$ with $5 \% \mathrm{CO}_{2}$. Hsa-miR-222-3p mimics, hsa-miR222-3p inhibitor, pcDNA3.1/Gas5 plasmid and negative control miRNA (miR-nc) were chemically synthesized by Shanghai Integrated Biotech Solutions Co., Ltd. (Shanghai, China).

\section{Transient transfection}

Cells were seeded in 6-well dishes the night before to give $80 \sim 90 \%$ confluence for plasmid and $40 \sim 50 \%$ for miRNA at the day of transfection. The following day cells were transfected with miR-222-3p mimics (50 nM), miR222-3p inhibitor (100 nM), miR-nc (50 nM), inhibitor-nc (100 nM), pcDNA3.1/Gas5 (4.0 $\mu \mathrm{g})$ or pcDNA3.1 (4.0 $\mu \mathrm{g})$ respectively using Lipofectamine 2000 (invitrogen), according to the manufacturer's instructions. After transfection for 6 hours, the medium was replaced with normal culture medium.

\section{RNA extraction and qRT-PCR analyses}

Total RNA extraction was conducted using a TRIzol reagent kit (Life Technologies, Inc., Rockville, MD, USA) according to the manufacture. Then, total RNA was reversely transcribed to complementary DNA

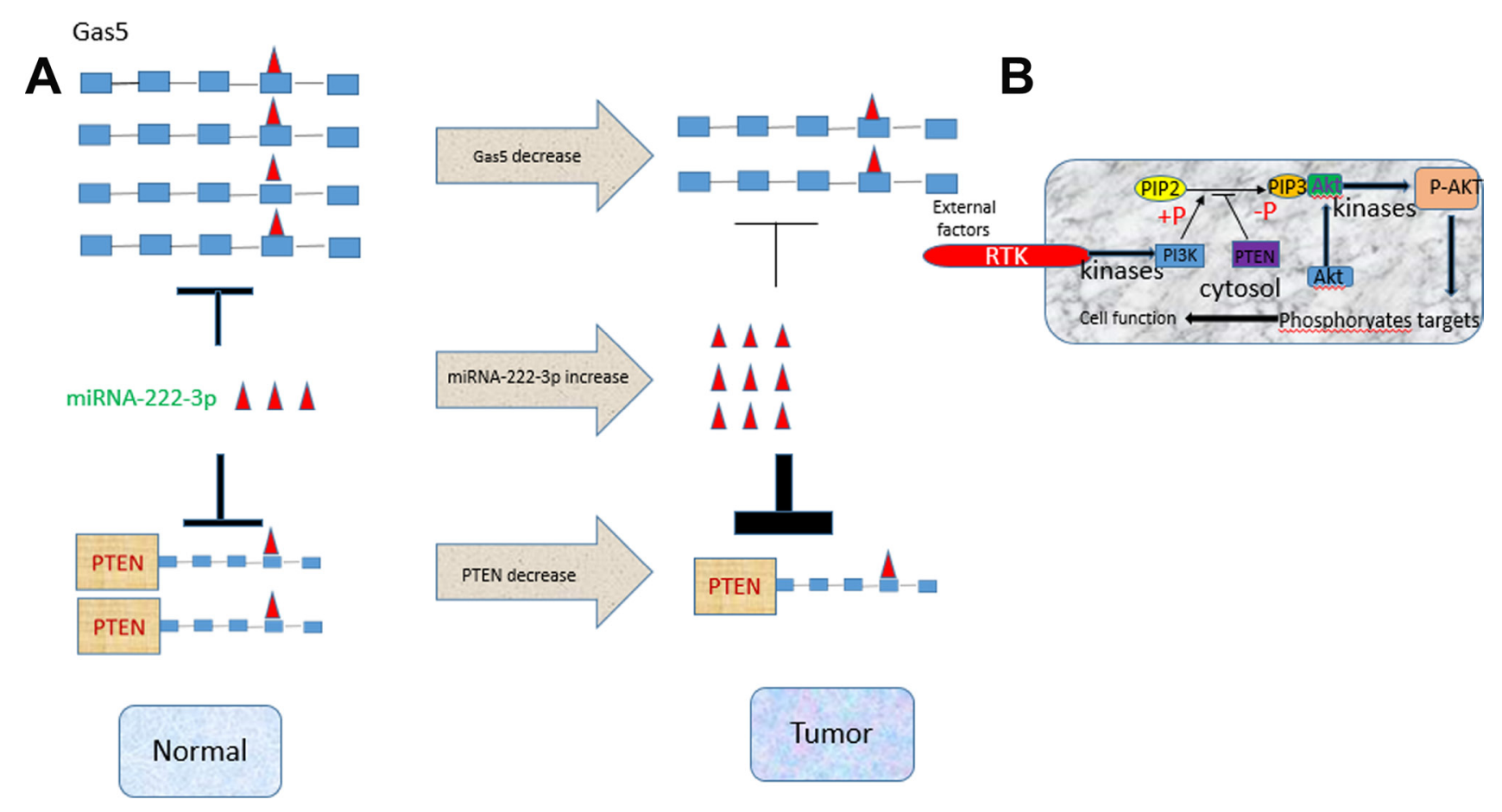

Figure 6: An illustrative figure detailing the relationship among Gas5, miR-222-3p and PTEN. (A) High expression of both Gas5 and PTEN, low expression of miR-222-3p in normal cells. In tumor cells low expression of Gas sponges less miR-222-3p that is highly expressed leading to a stronger inhibition of miR-222-3p on PTEN. (B) A detailed description on the PTEN/AKT-PTEN signaling pathway. Low expression of PTEN dephosphorylates less PIP3, resulting in an enhanced level of phosphorylation of AKT. 
(cDNA) using a reverse transcription kit (Thermo). QRT-PCR reactions were performed with EvaGreen $2 \times$ qPCR (abm) using LightCycler ${ }^{\circledR} 96$ (Roche). The relative expression of Gas5 mRNA and PTEN mRNA was calculated using the $2-\Delta \mathrm{CT}$ method normalized to GAPDH. The reaction conditions of PCR were as follows: $95^{\circ} \mathrm{C}$ pre-denaturation for 2 min followed by 40 cycles of $95^{\circ} \mathrm{C}$ denaturation for $15 \mathrm{~s}, 60^{\circ} \mathrm{C}$ annealing for $30 \mathrm{~s}$, and $72^{\circ} \mathrm{C}$ extension for $30 \mathrm{~s}$. For miR-222-3p expression detection, Stem-loop qRT-PCR Detection Kit (GenePharma) was used and U6 snoRNA was validated as the normalizer. Primer sequences were as follows: Gas5 forward 5'-AGCTGGAAGTTGAAATGG-3' and reverse 5'-CAAGCCGACTCTCCATA C-3', PTEN forward 5'-TGGAAAGGGACGAACTGGTG-3', reverse 5'-CATAGCGCCTCTGA CTGGGA-3', GAPDH forward 5'-ACCCACTCCTCCACCTTT-3', reverse 5'-GCTGTAG CCAAATTCGTTGT-3'.

\section{Cell proliferation assay}

Transfected PTC cells were seeded into 96-well plates in a density of $5 \times 10^{3}$ cells per well. At $24,48,72$ and $96 \mathrm{~h}$, culture medium was replaced with fresh medium containing MTT dye (20 $\mu$ l per well, Solarbio, Beijing) was added to each well and incubated $4 \mathrm{~h}$ at $37^{\circ} \mathrm{C}$. After removing the medium, dimethyl sulfoxide (DMSO) (150 $\mu \mathrm{l}$ per well; Sigma, USA) was added and mixed for $10 \mathrm{~min}$. The absorbance was detected by Universal Microplate Spectrophotometer (Bio-Tek Instruments, Inc., Winooski, VT, USA). OD570nm value was measured.

\section{Colony formation assay}

Seventy-two hours after transfection, cells were trypsinized (Solarbio, Beijing), replaced in 6-well plates at 200 cells per well and cultured in RPMI 1640 (Invitrogen) supplemented with $10 \%$ fetal bovine serum (Gibico) under routine conditions for 21 days. Medium was changed if needed. Then colonies were fixed with methanol and stained with $0.5 \%$ crystal violet (Sigma). Colonies were counted. Visible colonies were manually counted.

\section{Tumor formation assay}

Female BALB/c nude mice were purchased at 5-6 weeks of age from Beijing Vital River Laboratory Animal Technology Co., Ltd. For xenograft models, $5 \times 10^{5}$ BHP5-16 cells transfected with pcDNA3.1/Gas5 or pcDNA3.1 were subcutaneously injected in the bottom right back of BALB/c nude mice respectively. Tumor growth was examined every 3 days when the implantations were starting to grow bigger. After 6 weeks the mice were sacrificed, necropsies were performed and the tumor tissues were harvested. Tumor volumes were calculated as described previously.

\section{Dual-luciferase assay}

Cells $\left(2.0 \times 10^{4}\right)$ grown in a 96-well plate were cotransfected with $150 \mathrm{ng}$ of either MiR-nc or miR-222-3p mimics, 50 ng of psi-check Luciferase Expression Reporter (Promega) comprising 3'UTR of Gas5 (wild type or mutant type) for testing the relationship between miR222-3p mimics and Gas5 plasmid. Cells co-tansfected with $150 \mathrm{ng}$ of miR-222-3p mimics, $50 \mathrm{ng}$ of pEZX-MT01 Luciferase miRNA Expression Reporter (GeneCopeia) comprising 3 'UTR of PTEN (wild type or mutant type) were used for evaluation the relationship between miR222-3p and PTEN. In addition, cells co-tansfected with $150 \mathrm{ng}$ of miR-222-3p mimics, $25 \mathrm{ng}$ of pEZX-MT01 Luciferase miRNA Expression Reporter (GeneCopeia) comprising 3'UTR of PTEN (wild type), $75 \mathrm{ng}$ of Gas5 plasmid or empty vector were used to determine the relationship among miR-222-3p, PTEN and Gas5. Cells were harvested $48 \mathrm{~h}$ after transfection for luciferase assay using a luciferase assay kit (Promega) on a Synergy 2 Microplate Reader Fluorometer (BioTek) according to the manufacturer's instruction. The relative luciferase activity was calculated as the ratio of firefly luciferase activity versus renilla luciferase activity.

\section{Western blot}

Western blot analysis were conducted following standard protocols as described earlier [42]. Briefly, whole cell lysates were prepared and protein concentrations were quantified colourimetrically using a BCA Protein Assay Kit (Solarbio, Beijing). Samples were separated in a $10 \%$ SDS-polyacrylamide gel (Solarbio, Beijing) and blotted onto a PVDF membrane (Millipore). Immunoblot was performed with commercially available antibodies (anti PTEN antibody, 1:5000, Santa Cruz; anti- AKT antibody, 1:1000, BD Bioscience; anti-PAKT antibody, 1:1000, Cell Signaling Technology; antiGAPDH antibody, 1:5000, Santa Cruz.). ECL (Millipore) was applied for chemiluminescence detection. Immunoblot signal quantifications were performed using Image J software.

\section{Statistical analysis}

All data were expressed as mean \pm standard from three independent experiments. The independent experiments need to be at least three times. Statistical analysis was performed using ANOVA with SPSS 19.0 software (IBM Corporation, Armonk, NY, USA). The $P<0.05$ were considered statistically significant.

\section{ACKNOWLEDGMENTS}

The authors would like to thank the members of the Research Laboratory of Iodine and Thyroid Disease of 
Institute of Endocrinology, Metabolic Diseases Hospital, Tianjin Medical University.

\section{CONFLICTS OF INTEREST}

The authors declare no conflicts of interest in this work.

\section{GRANT SUPPORT}

This work was supported by the National Natural Science Foundation of China (No.81001226, No.30700689).

\section{REFERENCES}

1. Blomberg M, Feldt-Rasmussen U, Andersen KK, Kjaer SK. Thyroid cancer in Denmark 1943-2008, before and after iodine supplementation. Int J Cancer. 2012; 131:2360-6. https://doi.org/10.1002/ijc.27497.

2. Albores-Saavedra J, Henson DE, Glazer E, Schwartz AM. Changing patterns in the incidence and survival of thyroid cancer with follicular phenotype - papillary, follicular, and anaplastic: a morphological and epidemiological study. Endocr Pathol. 2007; 18:1-7.

3. Wang Y, Wang W. Increasing incidence of thyroid cancer in Shanghai, China, 1983-2007. Asia Pac J Public Health. 2015; 27:Np223-9. https://doi. org/10.1177/1010539512436874.

4. Gimm O. Thyroid cancer. Cancer Lett. 2001; 163:143-56.

5. Hundahl SA, Fleming ID, Fremgen AM, Menck HR. A National Cancer Data Base report on 53,856 cases of thyroid carcinoma treated in the U.S., 1985-1995 [see commetns]. Cancer. 1998; 83:2638-48.

6. Martin L, Chang HY. Uncovering the role of genomic "dark matter" in human disease. J Clin Invest. 2012; 122:1589-95. https://doi.org/10.1172/jci60020.

7. Shi X, Sun M, Liu H, Yao Y, Song Y. Long non-coding RNAs: a new frontier in the study of human diseases. Cancer Lett. 2013; 339:159-66. https://doi.org/10.1016/j. canlet.2013.06.013.

8. Yuan J, Yue H, Zhang M, Luo J, Liu L, Wu W, Xiao T, Chen X, Chen X, Zhang D, Xing R, Tong X, Wu N, et al. Transcriptional profiling analysis and functional prediction of long noncoding RNAs in cancer. Oncotarget. 2016; 7:8131-42. https://doi.org/10.18632/oncotarget.6993.

9. Liu XH, Liu ZL, Sun M, Liu J, Wang ZX, De W. The long non-coding RNA HOTAIR indicates a poor prognosis and promotes metastasis in non-small cell lung cancer. BMC Cancer. 2013; 13:464. https://doi. org/10.1186/1471-2407-13-464.

10. Shi X, Sun M, Liu H, Yao Y, Kong R, Chen F, Song Y. A critical role for the long non-coding RNA GAS5 in proliferation and apoptosis in non-small-cell lung cancer.
Mol Carcinog. 2015; 54:E1-e12. https://doi.org/10.1002/ mc. 22120 .

11. Li W, Li N, Shi K, Chen Q. Systematic review and metaanalysis of the utility of long non-coding RNAGAS5 as a diagnostic and prognostic cancer biomarker. Oncotarget. 2017; 8:66414-25. https://doi.org/10.18632/oncotarget. 19040.

12. Smith CM, Steitz JA. Classification of gas 5 as a multismall-nucleolar-RNA (snoRNA) host gene and a member of the 5'-terminal oligopyrimidine gene family reveals common features of snoRNA host genes. Mol Cell Biol. 1998; 18:6897-909.

13. Schneider C, King RM, Philipson L. Genes specifically expressed at growth arrest of mammalian cells. Cell. 1988; 54:787-93.

14. Salmena L, Poliseno L, Tay Y, Kats L, Pandolfi PP. A ceRNA hypothesis: the Rosetta Stone of a hidden RNA language? Cell. 2011; 146:353-8. https://doi.org/10.1016/j. cell.2011.07.014.

15. Li J, Yen C, Liaw D, Podsypanina K, Bose S, Wang SI, Puc J, Miliaresis C, Rodgers L, McCombie R, Bigner SH, Giovanella BC, Ittmann M, et al. PTEN, a putative protein tyrosine phosphatase gene mutated in human brain, breast, and prostate cancer. Science. 1997; 275:1943-47.

16. Tamura M, Gu J, Matsumoto K, Aota S, Parsons R, Yamada KM. Inhibition of cell migration, spreading, and focal adhesions by tumor suppressor PTEN. Science. 1998; 280:1614-7.

17. Song MS, Salmena L, Pandolfi PP. The functions and regulation of the PTEN tumour suppressor. Nat Rev Mol Cell Biol. 2012; 13:283-96. https://doi.org/10.1038/ nrm3330.

18. Li L, Wang Z, Hu X, Wan T, Wu H, Jiang W, Hu R. Human aortic smooth muscle cell-derived exosomal miR-221/222 inhibits autophagy via a PTEN/AKT signaling pathway in human umbilical vein endothelial cells. Biochem Biophys Res Commun. 2016; 479:343-50. https://doi.org/10.1016/j. bbrc.2016.09.078.

19. Monsalves E, Juraschka K, Tateno T, Agnihotri S, Asa SL, Ezzat S, Zadeh G. The PI3K/AKT/mTOR pathway in the pathophysiology and treatment of pituitary adenomas. Endocr Relat Cancer. 2014; 21:R331-44. https://doi. org/10.1530/erc-14-0188.

20. Trivellin G, Butz H, Delhove J, Igreja S, Chahal HS, Zivkovic V, McKay T, Patocs A, Grossman AB, Korbonits M. MicroRNA miR-107 is overexpressed in pituitary adenomas and inhibits the expression of aryl hydrocarbon receptor-interacting protein in vitro. Am J Physiol Endocrinol Metab. 2012; 303:E708-19. https://doi. org/10.1152/ajpendo.00546.2011.

21. Liz J, Esteller M. IncRNAs and microRNAs with a role in cancer development. Biochim Biophys Acta. 2016; 1859:169-76. https://doi.org/10.1016/j.bbagrm.2015.06.015. 
22. Rosignolo F, Memeo L, Monzani F, Colarossi C, Pecce V, Verrienti A, Durante C, Grani G, Lamartina L, Forte S, Martinetti D, Giuffrida D, Russo D, et al, and Rignolo F1. MicroRNA-based molecular classification of papillary thyroid carcinoma. Int J Oncol. 2017; 50:1767-77.

23. Saiselet M, Gacquer D, Spinette A, Craciun L, DecaussinPetrucci M, Andry G, Detours V, Maenhaut C. New global analysis of the microRNA transcriptome of primary tumors and lymph node metastases of papillary thyroid cancer. BMC Genomics. 2015; 16:828. https://doi.org/10.1186/ s12864-015-2082-3.

24. Fang L, Li H, Wang L, Hu J, Jin T, Wang J, Yang BB. MicroRNA-17-5p promotes chemotherapeutic drug resistance and tumour metastasis of colorectal cancer by repressing PTEN expression. Oncotarget. 2014; 5:2974-87. https://doi.org/10.18632/oncotarget.1614.

25. Garofalo M, Di Leva G, Romano G, Nuovo G, Suh SS, Ngankeu A, Taccioli C, Pichiorri F, Alder H, Secchiero P, Gasparini P, Gonelli A, Costinean S, et al. miR-221\&222 regulate TRAIL resistance and enhance tumorigenicity through PTEN and TIMP3 downregulation. Cancer Cell. 2009; 16:498-509. https://doi.org/10.1016/j.ccr.2009.10.014.

26. Chun-Zhi Z, Lei H, An-Ling Z, Yan-Chao F, Xiao Y, Guang-Xiu W, Zhi-Fan J, Pei-Yu P, Qing-Yu Z, ChunSheng K. MicroRNA-221 and microRNA-222 regulate gastric carcinoma cell proliferation and radioresistance by targeting PTEN. BMC Cancer. 2010; 10:367. https://doi. org/10.1186/1471-2407-10-367.

27. Pickard MR, Williams GT. Molecular and Cellular Mechanisms of Action of Tumour Suppressor GAS5 LncRNA. Genes (Basel). 2015; 6:484-99. https://doi. org/10.3390/genes6030484.

28. Qiao HP, Gao WS, Huo JX, Yang ZS. Long non-coding RNA GAS5 functions as a tumor suppressor in renal cell carcinoma. Asian Pac J Cancer Prev. 2013; 14:1077-82.

29. Liu Z, Wang W, Jiang J, Bao E, Xu D, Zeng Y, Tao L, Qiu J. Downregulation of GAS5 promotes bladder cancer cell proliferation, partly by regulating CDK6. PLoS One. 2013; 8:e73991. https://doi.org/10.1371/journal. pone.0073991.

30. Lu X, Fang Y, Wang Z, Xie J, Zhan Q, Deng X, Chen H, Jin J, Peng C, Li H, Shen B. Downregulation of gas5 increases pancreatic cancer cell proliferation by regulating CDK6. Cell Tissue Res. 2013; 354:891-6. https://doi.org/10.1007/ s00441-013-1711-x.

31. Pickard MR, Williams GT. Regulation of apoptosis by long non-coding RNA GAS5 in breast cancer cells: implications for chemotherapy. Breast Cancer Res Treat. 2014; 145:359-70. https://doi.org/10.1007/s10549-014-2974-y.

32. Guo LJ, Zhang S, Gao B, Jiang Y, Zhang XH, Tian WG, Hao S, Zhao JJ, Zhang G, Hu CY, Yan J, Luo DL. Low expression of long non-coding RNA GAS5 is associated with poor prognosis of patients with thyroid cancer. Exp Mol Pathol. 2017; 102:500-04. https://doi.org/10.1016/j. yexmp.2017.05.008.
33. Zeng LP, Hu ZM, Li K, Xia K. miR-222 attenuates cisplatin-induced cell death by targeting the PPP2R2A/ AKT/mTOR Axis in bladder cancer cells. J Cell Mol Med. 2016; 20:559-67. https://doi.org/10.1111/jcmm.12760.

34. Chen WX, Hu Q, Qiu MT, Zhong SL, Xu JJ, Tang JH, Zhao JH. miR-221/222: promising biomarkers for breast cancer. Tumour Biol. 2013; 34:1361-70. https://doi.org/10.1007/ s13277-013-0750-y.

35. Marchetti C, Palaia I, Giorgini M, De Medici C, Iadarola R, Vertechy L, Domenici L, Di Donato V, Tomao F, Muzii L, Benedetti Panici P. Targeted drug delivery via folate receptors in recurrent ovarian cancer: a review. Onco Targets Ther. 2014; 7:1223-36. https://doi.org/10.2147/ott. s40947.

36. Gits CM, van Kuijk PF, Jonkers MB, Boersma AW, van Ijcken WF, Wozniak A, Sciot R, Rutkowski P, Schoffski P, Taguchi T, Mathijssen RH, Verweij J, Sleijfer S, et al. MiR-17-92 and miR-221/222 cluster members target KIT and ETV1 in human gastrointestinal stromal tumours. Br J Cancer. 2013; 109:1625-35. https://doi.org/10.1038/bjc.2013.483.

37. Ihle MA, Trautmann M, Kuenstlinger H, Huss S, Heydt C, Fassunke J, Wardelmann E, Bauer S, Schildhaus HU, Buettner R, Merkelbach-Bruse S. miRNA-221 and miRNA222 induce apoptosis via the KIT/AKT signalling pathway in gastrointestinal stromal tumours. Mol Oncol. 2015; 9:1421-33. https://doi.org/10.1016/j.molonc.2015.03.013.

38. Pallante P, Visone R, Ferracin M, Ferraro A, Berlingieri MT, Troncone G, Chiappetta G, Liu CG, Santoro M, Negrini M, Croce CM, Fusco A. MicroRNA deregulation in human thyroid papillary carcinomas. Endocr Relat Cancer. 2006; 13:497-508. https://doi.org/10.1677/erc.1.01209.

39. Keutgen XM, Filicori F, Crowley MJ, Wang Y, Scognamiglio T, Hoda R, Buitrago D, Cooper D, Zeiger MA, Zarnegar R, Elemento O, Fahey TJ 3rd. A panel of four miRNAs accurately differentiates malignant from benign indeterminate thyroid lesions on fine needle aspiration. Clin Cancer Res. 2012; 18:2032-8. https://doi.org/10.1158/10780432.ccr-11-2487.

40. Yip L, Kelly L, Shuai Y, Armstrong MJ, Nikiforov YE, Carty SE, Nikiforova MN. MicroRNA signature distinguishes the degree of aggressiveness of papillary thyroid carcinoma. Ann Surg Oncol. 2011; 18:2035-41. https://doi.org/10.1245/s10434-011-1733-0.

41. Lee JC, Zhao JT, Clifton-Bligh RJ, Gill A, Gundara JS, Ip JC, Glover A, Sywak MS, Delbridge LW, Robinson BG, Sidhu SB. MicroRNA-222 and microRNA-146b are tissue and circulating biomarkers of recurrent papillary thyroid cancer. Cancer. 2013; 119:4358-65. https://doi.org/10.1002/ cncr.28254.

42. Liu J, Xu J, Li H, Sun C, Yu L, Li Y, Shi C, Zhou X, Bian X, Ping Y, Wen Y, Zhao S, Xu H, et al. miR-146b-5p functions as a tumor suppressor by targeting TRAF6 and predicts the prognosis of human gliomas. Oncotarget. 2015; 6:29129-42. https://doi.org/10.18632/oncotarget.4895. 